\title{
El azar y la finalidad (Estudio antropológico)
}

El empirismo moderno reanuda los ataques contra el principio de causalidad, pero hay muchos filósofos que lo consideran como evidente e irreductible a todo otro principio. De todas formas hay que decir que el principio de causalidad debe ser reducido a la percepción del ser.

Los científicos modernos cuando niegan el que se dé una rigurosa causalidad en la naturaleza, quieren significar con esas palabras que no se da un «determinismo riguroso».

Los científicos de los siglos XIX y XX también dieron su opinión sobre el azar aunque originariamente todas esas ideas procedan de Aristóteles, Boecio, santo Tomás y otros.

La ruptura más radical con la visión mecanicista del mundo sustentada por la ciencia de nuestra época la representa la teoría de la sincronicidad debida a Jung Pauli, aunque varios físicos marcharon por ese camino.

Las teorias clásicas sobre la percepción extrasensorial propuesta por Carington, Tyrel, Hardy y otros eran variaciones sobre un mismo tema. Los biólogos han tomado interés por las potencialidades de la materia viva en cuanto a edificar formas de complejidad superior que presentan la unidad en la diversidad sobre un nivel más alto. Este interés lo comparte el profesor Rodríguez Villanueva con las últimas teorías aparecidas. Aquí nos paramos a analizar la de Oparin defendida, al parecer por gran parte de los biólogos y que en síntesis viene a decir que la vida se originó por azar.

\section{El empirismo, el azar y el principio de causalidad}

Los filósofos modernos mostraron escasa inclinación a ocuparse del problema del azar, pero el empirismo moderno, que en más de un punto depende del nominalismo, reanudará los ataques de éste contra el principio de causalidad.

Juan Locke (1632-1704), que recibió una formación nominalista, conservó, sin embargo, el principio de causalidad en esta forma: de nada no deviene 
nada, ex nihilo nihil fit ${ }^{1}$. Pero David Hume (1711-1776) llevó el empirismo hasta el extremo y negó que los principios universales pudieran ser leyes objetivas necesarias. Rechazó especialmente el principio de causalidad ${ }^{2}$.

Según Hume la experiencia nos muestra hechos sucesivos, no causas que produzcan efectos. Por otra parte, nos formamos de la causa y del efecto representaciones distintas $\mathrm{y}$, por tanto, separables; ¿por qué, pues; sería contradictorio que éste existiera sin aquélla? Es preciso, sin embargo, explicar cómo llegan los hombres a admitir el principio de causalidad.

La explicación es de orden psicológico: dos hechos que se suceden regularmente nos producen la costumbre de verlos juntos; tan pronto como vemos uno, esperamos otro, y traducimos esta impresión diciendo que el uno lleva consigo el otro, que el primero causa el segundo.

El principio de causalidad tiene, pues, un carácter de necesidad, pero de orden psicológico. Indudablemente con esto no se explica por qué no todas las sucesiones son consideradas como series causales (por ejemplo, el día sucede a la noche, sin ser efecto de ella). Pero lo cierto es que la teoría de Hume fue recogida por los empiristas del siglo XIX, especialmente por John Stuart Mill ${ }^{3}$.

J. Stuart Mill (1806-1873) en la primera edición del $A$ System of Logic Raciocinative and inductive escribe:

Un acontecimiento que sucede por azar podría describirse mejor como una coincidencia de la cual no tenemos fundamento para inferir una uniformidad... Los hechos casualmente juntados son separadamente efectos de causas... pero de causas diferentes, de causas no unidas por una ley ${ }^{4}$.

En el criticismo de Kant, el principio de causalidad recibió una significación nueva: fue declarado necesario, con necesidad subjetiva, porque es una forma a priori del pensamiento. Esta explicación ha sido aceptada por numerosos filósofos.

Henry Bergson no atribuye al principio de causalidad más que una necesidad subjetiva de orden pragmático. Es un principio concebido por la inteligencia. Ahora bien, ésta no se detiene en el conocimiento adecuado de la realidad porque es movida por los intereses de la vida y persigue fines útiles.

Para guiar la acción, la inteligencia inmoviliza y fracciona, en sus representaciones y en sus ideas, el deslizamiento perpetuo de la vida y de toda realidad, y se vale de diversos procedimientos, especialmente del principio de causalidad, para darnos una apariencia de actividad y de vida.

1. LOCKE, J., Essay II, 26,1.

2. HumE, D., Treatise of human Nature I, 3, sec. 3.

3. Stuart Mill, J., System of logic ratiocinative and inductive 1, I, c. $3 \S \S 6,7,8$, Londres 1843.

4. StUART MILl, J., System of logic ratiocinative and inductive, Logmans-Green, Londres $1947,1.3$ c. $17 \& 2$, p. 345. 
Los neoescolásticos han estado siempre de acuerdo en admitir la necesidad absoluta del principio de causalidad, pero van por caminos divergentes, tan pronto como se trata de justificar su modo de ver.

Estas divergencias se han manifestado en numerosas controversias, que tuvieron lugar especialmente a consecuencia de una comunicación de Amédée de Margerie (1825-1905) al Congreso Científico Internacional de los Católicos, celebrado en París en 1888; posteriormente en torno a un artículo de Jacq. Laminne (1864-1924) aparecido en 1912 en la Revue Néoscolastique de Philosophie, de Lovaina 5; asimismo, después de 1915, con ocasión de una obra de Gașpar Isencrahe (1844-1921), que ponía en duda el valor del principio de causalidad ${ }^{6}$. Esta última controversia se desarrolló principalmente en Alemania, aunque naturalmente también repercutió en otros países.

Son numerosos los que consideran el principio de causalidad como inmediatamente evidente e irreductible a todo otro principio. En este caso, la explicación de la realidad reposa en un doble fundamento, el principio de contradicción (o el de identidad) y el principio de causalidad: mientras que éste puede ser negado sin contradicción formal, el primero no puede ser rechazado sin arruinar totalmente el edificio intelectual y, por consiguiente, el principio de causalidad ${ }^{7}$.

Jos. Geyser (1869) apela al método fenomenológico para resolver este problema. A la luz de la conciencia, vemos «surgir» en nosotros actos de inteligencia y de voluntad. Reflexionando sobre estos hechos concretos (y sin hacer un análisis de conceptos), podemos percibir, según Geyser, el vínculo esencial: pertenece a la esencia de «lo que comienza» a encontrarse en relación de dependencia frente a una causa, de suerte que es imposible comenzar a ser si no es dependiendo de una causa ${ }^{8}$. niones?

¿Cómo enjuiciar estas diferencias? ¿Cómo enjuiciar estas diferentes opi-

No es posible fundamentar la necesidad absoluta del principio de causalidad sobre una base puramente inductiva. Sin embargo, es cierto que su justificación no puede establecerse más que en conexión con la experiencia, porque sólo en una experiencia que nos pone en contacto con el «ser» concreto y real percibimos el valor de ser; ahora bien, este valor de ser es el alma de toda ver-

5. RAEYMAeker, L., Filosofía del ser (versión de María Dolores Mouton y Valentín García Yebra), B.H.F., Gredos, Madrid 1961 (2. ${ }^{\text {a }}$ ed. revisada), p. 289.

6. ISENCRAHE, G., Ueber die Grundlegung eines bundigen kosmologischen Gottesbeweises, Kempten, Munich 1915.

7. Esta opinión es sostenida por Lorenz Fuetscher (1894-1935), Pedro Descoqs (18771946), J. de Vries, etc.

8. La descripción fenomenológica de Geiser se refiere a la actividad espiritual e inmanente del hombre, que no se debe confundir con la causalidad (externa). Como la actividad humana es siempre a la vez transitiva e inmanente, implica siempre este factor de causalidad. 
dad absoluta, y, por consiguiente, también el principio de causalidad debe ser reducido a la percepción del ser.

La tesis que pretende que este principio no puede deducirse del principio de identidad o de contradicción es verdadera en el caso de que uno quiera atenerse al plano de los conceptos «abstractos». En efecto, la definición abstracta de la quididad específica o genérica (por ejemplo, el hombre es un animal racional) está, por decirlo así, cerrada, en el sentido de que no menciona ningún otro ser.

Todo ser está determinado en su naturaleza; por consiguiente lo está también en su actividad, cuyo principio substancial es la naturaleza. Si la acción está determinada, el efecto lo estará también. Esto quiere decir que la acción está determinada en sí misma, en razón de su orientación a un término determinado. Este término es, pues, la razón de la acción.

El efecto no es, pues, únicamente el término final, el «remate» de la operación; es, sobre todo, el término que hay que alcanzar, la meta final; la operación apunta a él, lo persigue, tiende a él, y no está determinada en sí misma sino en virtud de este fin, al cual está ordenada y por el cual es atraída: la operación depende del fin; la eficiencia encuentra su razón, su sentido, en la meta perseguida. La finalidad, el influjo del fin sobre la operación, es un elemento esencial de la causalidad: quidquid agit, agit propter finem ${ }^{9}$.

La ley de la finalidad va unida a la de la eficiencia; una y otra anuncian aspectos de una misma actividad causal. El efecto es un producto, depende de la operación; pero la operación tiende al efecto, se refiere a $\dot{\varepsilon}, y$, por consiguiente, depende de él. Hay un influjo recíproco del efecto soure la causa (en el orden de la finalidad) y de la causa sobre el efecto (en el orden de la eficiencia). La una no se da sin el otro: no hay eficiencia sin finalidad, ni finalidad sin eficiencia. Este influjo reciproco conjuntamente forman la relación causal.

Toda causalidad material se apoya necesariamente en una actividad intelectual que la ordena y guía: intellectus est ordinare. El mundo material postula una o varias inteligencias que orienten sus operaciones hacia un fin determ: nado.

El hombre, en cuanto que es espiritual y subsistente, conoce y persigue su propio bien; tiende a un bien que le es personal. Por otra parte, el hombre es material, forma parte del universo. Pero, gracias a su actividad espiritual, posee cierto dominio de su cuerpo, lo cual le permite ejercer una acción personal sobre las cosas circundantes.

A la finalidad interna de las cosas viene a añadirse, de esta manera, una

9. Garrigou-LAgrange, R., Le realisme du principe de finalité, Bibliot. franç. de phii. $2^{e}$ série, Paris 1932, pp. 95-121. 
finalidad externa, que se apoya en la primera, pues la utiliza, y está vinculada a una finalidad interna personal.

Muchas veces, cuando los científicos modernos niegan el principio de causalidad o la existencia de causas, tienen para ellos esas expresiones distinto significado del que se da en la filosofía tradicional. Unas veces toman por Principio de Causalidad el que se pudiera llamar principio del determinismo y se enuncia: En las mismas condiciones, las mismas causas producen los mismos efectos.

Otras veces toman por principio de previsibilidad, para el que Heisenberg propone el enunciado: Si de un sistema aislado se conoce el estado presente en todos sus detalles, es posible calcular el estado futuro.

Cuando niegan que se dé una rigurosa causalidad en la naturaleza, quieren significar con esas palabras que no se da un «determinismo riguroso». La confusión nace de la manera de hablar. Ellos de hecho admiten la existencia de causas en los fenómenos, y las buscan con estudios e investigaciones, aunque tal vez no hablen explícitamente de ellas.

El tema del azar está de moda por la controversia acerca del sentido del principio de indeterminación de Heisenberg sobre la imposibilidad de conocer la trayectoria de los electrones: unos lo consideran válido sólo si se refiere al estado actual de la ciencia; otros lo interpretan como un límite intrínsecamente insuperable para el conocimiento científico; otros, en fin, le dan un alcance ontológico, manteniendo que es la realidad misma la que en su estracto es indeterminada. Monod se adhiere a estos últimos, hablando de azar esencial.

\section{Los científicos del siglo $X I X$ y $X X$ y el azar}

En español, el azar, la casualidad, el acaso, resultan casi siempre sinónimos entre sí. Con frecuencia se lee en la prensa, a propósito de un incendio, que el siniestro parece casual, queriendo indicar así que no ha sido intencionado. Tal es la manera corriente de hablar.

Se oye con frecuencia la palabra azar en los labios de los jugadores o de los que ponen su esperanza en un billete de la lotería: he tenido suerte, es una feliz casualidad, exclama el ganador. Y esto significa solamente que el resultado que le llena de satisfacción no podía ser ni querido con eficacia ni previsto con seguridad.

El científico, según F. Gregoire, reserva en general la palabra azar para aquellos sucesos que son el resultado de la unión de muchísimos determinismos muy complejos y cuyo tipo es la salida de un número de la lotería o en la ruleta. Esos sucesos cuyas causas no son todas conocidas o bien desconocidas, o son tan múltiples que desbordan nuesta posibilidad de abarcarlas, se dicen que están regidos por el azar. 
En parecidos términos dice Borel:

La característica de los fenómenos que llamamos fortuitos, o debidos al azar, es la de depender de causas demasiado complejas para que podamos conocerlas y estudiarlas ${ }^{10}$.

Para Laplace, el azar es tan sólo una palabra que encubre nuestra ignorancia.

Cien años más tarde $\mathrm{H}$. Poincaré reproduciría la misma idea, al recoger el sentir de los científicos contemporáneos suyos, de su época, en aquella frase:

El azar no es más que la medida de nuestra ignorancia ${ }^{11}$.

Para Poincaré como para Laplace el verdadero azar no existe: si en ciertos fenómenos hay un azar aparente, esta apariencia es debida, ya a nuestra impotencia para resolver un problema demasiado arduo para las fuerzas de nuestro espíritu, ya a nuestra ignorancia de los datos necesarios para su solución. Así, pues, el término azar designaría simplemente la resultante de procesos muy numerosos, muy complejos, muy mal conocidos... para que se la pueda calcular de antemano.

A partir del siglo XIX abundaron los análisis sobre el concepto de azar y así vemos que Charles Sanders Peirce (1839-1914) considera el tychismo (afirmación del azar) como una de las grandes categorías cosmológicas, junto al synechismo (afirmación de la continuidad) y el agapismo (afirmación de la evolución) ${ }^{12}$.

El tychismo, o tiquismo castellanizándolo, es la doctrina que resulta de una de las tres grandes categorías cosmológicas: la categoría del azar. Esta categoría está relacionada con la categoría metafísica que se refiere a los modos de existencia, particularmente al modo llamado justamente así, «azar».

Peirce admite, o postula, un "puro azar», el azar engendra, a su entender, hábitos y regularidades. Ello se debe probablemente a que hay continuidad, por lo menos «continuidad evolutiva». Por eso Peirce estima que el tiquismo no es en modo alguno incompatible con el sinequismo, antes bien, se halla estrictamente correlacionado con él.

En un sentido más general el tiquismo es la doctrina o,-más ampliamente, la actitud filosófica que prefiere un mundo «espontáneo» y en constante «cre-

10. Borel, E., Le hasard, F. alcan, Paris 1932, p. 7.

11. Poincare, H., Ciencia y método (trad. de M. García Miranda), Espasa-Calpe, Buenos Aires 1944, p. 56.

12. Peirce, C.S., The Doctrine of Chances, Popular Science Monthly (marzo 1878), reimp. en Chance, Love and Logic, 1923, ed. M.R. Cohen, pp. 61-81. 
cimiento» a un mundo «determinado» y «terminado». Es la actitud que, junto a Peirce, mantuvieron Bergson ${ }^{13} \mathrm{y}$ William James ${ }^{14}$.

Antoine Agustin Cournot (1801-1877) ha mantenido una doctrina sobre el azar semejante a la aristotélica. Según Cournot hay dos tipos de series causales: las solidarias, que expresan el orden, y las independientes entre sí, que expresan el azar:

Los acontecimientos producidos por la combinación o coincidencia de otros acontecimientos pertenecientes a series independientes son los que se llaman acontecimientos fortuitos o resultados del azar ${ }^{15}$.

¿Qué es el azar para Cournot?:

Ningún fenómeno o suceso es producido sin causa ${ }^{16}$.

El clásico ejemplo de la teja que cae sobre un transeúnte aparece en su obra Materialisme, Vitalisme, Rationalisme. Se lo aplica a sí mismo, que iba a cumplir en el momento de la publicación setenta y cuatro años:

Una teja cae de un tejado, sea que yo pase, sea que no pase por la calle; no hay ninguna conexión, ninguna solidaridad, ninguna dependencia entre las causas que determinan la caída de la teja y las que me han hecho salir de mi casa para llevar una carta al correo. La teja me cae sobre la cabeza, y he ahí al viejo lógico puesto definitivamente fuera de servicio: es un encuentro fortuito que tiene lugar por azar...

Los hechos que suceden por azar o por combinación fortuita, bien lejos de eliminar la idea de casualidad, bien lejos de ser efectos sin causa, exigen para su producción el concurso de varias causas. El carácter de fortuito deriva sólo del carácter de independencia de las causas concurrentes ${ }^{17}$.

La independencia de que habla Cournot es la ausencia de relaciones que muestren en una de las series las causas o la razón de la otra. La serie de sucesos que constituye la caída de la teja no tiene por consecuencia ninguno de los

13. Bergson, H., La evolución creadora (trad. de M. ${ }^{a}$ Luisa Pérez Torres de la ed. or. L'évolution créatrice en Presses Universitaires de France, Paris 1969), col. Austral, EspasaCalpe, Madrid 1973, cap. III, pp. 170-239.

14. JAMES, W., Carta a Bergson del 13 de junio de 1907 (The Selected Letters of William James, ed. Elizabeth Hardwick, 1961, pp. 237-238.

15. COURNot, A., Essai sur les fundaments de nos connaissances et sur les caractères de là critique philosophique, Hachette, Paris 1922, n. 30, pp. 37-38.

16. Cournot, A., Exposition de la théorie des chances et des probabilités, Hachette, Paris 1843 , n. 39, p. 71 .

17. Cournot, A., Materialisme, Vitalisme, Rationalisme, Hachette, Paris 1923, sec. 4 \& 3 , p. 222. 
detalles del paseo del viejo lógico, e inversamente el paseo no envuelve consigo ninguna condición para la caída de la teja. Según Cournot:

lo que hay de fundamental y de categórico en la noción del azar es la idea de la independencia o de la solidaridad entre diversas series de causas ${ }^{18}$.

Claro está que además se distingue en el azar otro elemento el cual es positivo y es el concurso o el encuentro de tales series de causas, es decir, la interferencia, la intersección de ellas.

Los modernos presentan como de Cournot toda esta concepción del azar, pero parece que ya en 1714 Juan de la Placette nos habla de esta concepción y más atrás tenemos a san Francisco de Sales el cual ya escribía en el año 1616:

Estos casos fortuitos resultan por la concurrencia de varias causas que, sin tener un enlace natural unas con otras, produce cada una su efecto particular, de tal manera, que de su encuentro nace otro efecto de otra naturaleza, al cual, sin que se haya podido preverlo, todas estas causas diferentes han contribuido ${ }^{19}$.

En realidad todas estas ideas originariamente proceden de Aristóteles, prolongando la tradición peripatética Boecio, santo Tomás y tantos otros, y reproducen la fórmula y el ejemplo mismo propuestos por el Estagirita.

Por consiguiente, las ideas de Cournot se encuentran implícitas substancialmente en Aristóteles; pero Cournot las declara más, las presenta con brillantez y las elabora según el gusto moderno.

El azar parece implicar los siguientes elementos:

1) Hay causas o series causales relativamente independientes.

2) Tiene lugar un encuentro de ellas.

3) Sin que lo pretendan o algún hombre lo provoque.

4) En el lenguaje ordinario sólo se habla de causalidad cuando el resultado nos interesa o nos llama la atención; es algo que parece pretendido y preparado sin haberlo sido. Es decir, es una casualidad y no una causalidad.

Émile Boutroux (1845-1921) encuentra una insuficiencia radical en la afirmación del determinismo absoluto expresado en las leyes de la conservación de la substancia y de la energía.

La descripción reflexiva de lo real muestra la existencia de diversas capas ontológicamente irreductibles y muestra, además, la contingencia de cada capa respecto a la anterior ${ }^{20}$.

18. Cournot, A., Essai sur les fondaments... op. cit. p. 41.

19. SAles, San Francisco, Traité de l'amour de Dieu, 1. 2 c. 3.

20. Sciacca, M.F., La filosofía hoy (trad. de Claudio Matons Rossi y Juan José Ruiz Cuevas de La Filosofía oggi, Ed. Luis Miracle, Barcelona 1961, tomo I, p. 68. 
La conciencia es, pues, contingente con respecto a la vida; la vida, con respecto a la materia; la materia, con respecto a los géneros; los géneros, con respecto al ser; el ser, con respecto a la necesidad relativa; ésta, con respecto a la necesidad absoluta.

Según Émile Borel (1871-1956), la necesidad y el determinismo pueden ser afirmados de la realidad globalmente, pero no de las realidades en particular; cuanto más particular es una realidad, tanto más azarosa es. El azar puede tratarse mediante leyes estadísticas que tienden a convertirse, pero sin jamás lograrlo, en leyes absolutas. Borel indica que las leyes deterministas expresan el estado más probable ${ }^{2 !}$.

Borel decía que el azar es lo que está fuera de toda regla, de toda ley:

La experiencia de cada día nos enseña a desconfiar de las leyes a las cuales se pretenden someter los sucesos fortuitos. Sin embargo en una serie de pruebas repetidas un gran número de veces en las mismas condiciones, cada uno de los sucesos posibles se presenta con una frecuencia relativa que viene a ser igual a su probabilidad; la aproximación crece ordinariamente al crecer el número de pruebas ${ }^{22}$.

El teorema de Bernouilli es un reflejo de la ley del azar.

Por último para François Meyer, el universo en estado corpuscular, que obedece a leyes estadísticas de la mecánica cuántica, representa el estado de menor probabilidad; el universo formado representa el estado de mayor probabilidad $^{23}$.

A comienzos de este siglo, Lord Rutherford y el gran físico danés Niels Bohr concibieron un modelo de átomo inquietantemente simple, consistente en un sistema solar en miniatura; los electrones de carga negativa circulaban como planetas en torno a un núcleo positivamente cargado. Pero este modelo chocó con una paradoja tras otra: los electrones no se comportaban como los planetas; saltaban de una órbita a otra sin atravesar espacios intermedios, cual si la Tierra se transfiriera súbitamente a la órbita de Marte sin realizar viaje alguno. Las órbitas en sí, por otra parte, no eran trayectorias lineales, sino senderos amplios y difusos, y no tenía sentido preguntarse, por ejemplo, en qué punto de su órbita se encontraba el electrón del átomo de hidrógeno en un momento determinado del tiempo. Estaba en toda partes por igual.

De Broglie proclamó que el electrón es a la vez corpúsculo y vibración. Este dualismo, básico para la física moderna, fue denominado por Bohr, Principio de Complementariedad, y la escuela fundada por Bohr Escuela de

21. Borel, E., Le hasard, Alcan, Paris 1932 (traducción española El azar, 1948).

2.2. BOREL, E., Le hasard, op. cit., p. 214.

23. MEYER, F., L'acceleration evolutive, 1947 y Problématique de l'évolution, 1954. 
Copenhague que convirtió a la complementariedad en una especie de credo. Heisenberg comentó:

El concepto de complementariedad pretende describir una situación en la que podemos considerar a un mismo acontecimiento a través de dos marcos de referencia distintos. Estos dos marcos de referencia se excluyen mutuamente, pero también se complementan ${ }^{24}$.

La teoría de Adrian Dobbs contiene refinamientos que toman en cuenta la imprevisibilidad e indeterminación del futuro en la fiesta cuántica. La flecha del tiempo, avanzando en una segunda dimensión temporal, atraviesa un mundo probabilístico, no ya determinístico; y se parece más al frente de una ola que a la afilada punta de una flecha.

El interés mayor de la hipótesis de Dobbs radica en su intento de desarrollar una explicación física de la telepatía y la precognición más sofisticada que otras hipótesis.

En cuanto a la precognición, el quid de la cuestión radica en que la anticipación de los acontecimientos futuros actúa en la segunda dimensión temporal, donde las probabilidades objetivas juegan el mismo papel que las relaciones causales en la física clásica.

Dobbs utiliza el término pre-visión en lugar de precognición; lo hace para indicar que no se refiere a la profecía sino a la percepción de los factores probabilísticos existentes en un sistema, que le predisponen hacia un determinado estado futuro. El sujeto recibe información sobre estos factores a través de hipotéticos mensajeros que Dobbs denomina psitrones y que operarían en su segunda dimensión temporal.

El concepto de Dobbs sobre el psitrón es, de hecho un producto final de las tendencias actuales de la teoría cuántica y la investigación cerebral. La masa del psitrón es imaginaria (en el sentido matemático), y por lo tanto, conforme a la teoría de la relatividad, puede desplazarse más rápido que la luz, indefinidamente, sin pérdida de su momento imaginario ${ }^{25}$.

Dobbs no aborda directamente el problema mente-cuerpo; presupone que ciertos procesos cerebrales dan lugar a ciertos estados de conciencia; lo que ocurriría tanto cuando los procesos cerebrales en cuestión son inducidos por percepciones extrasensoriales como cuando responden a una sensación común.

La distancia espacial que debe recorrer el psitrón es insignificante. Llegamos así a la paradójica conclusión de que las teorías físicas como las de Adrian Dobbs, por ingeniosas que fueran, explicarían tan sólo el elemento «extra» de

24. Heisenberg, W., Der Teil und das Ganze, München 1969, p. 113.

25. KoestLer, A., Las raíces del azar (trad. de Rolando Hanglin), Ed. Kairós, Barcelona 1974, pp. 98-99. 
la percepción extrasensorial; sin desvelar el misterio básico de la percepción ordinaria sensorial.

La conciliación entre los mundos conceptuales de la parapsicología y la física moderna supone un paso importante hacia la demolición de la máxima superstición de nuestra era: el universo de relojería materialista concebido por la física del siglo XIX.

La ruptura más radical con la visión mecanicista del mundo sustentada por la ciencia de nuestra época, la representa la teoría de la sincronicidad, debida a Jung-Pauli.

Existió un precursor cuyas ideas ejercieron considerable influencia sobre Jung: el biólogo austriaco Paul Kammerer, que se suicidó en 1926 a los cuarenta y cinco años. Era lamarckiano y suponía que las habilidades y progresos físicos obtenidos por los padres eran hereditarios para sus descendientes.

Kammerer desarrolla su idea central de que con la causalidad coexiste un principio no-causal operante en el universo, que tiende a la unidad. Kammerer estaba particularmente interesado en las series temporales de acontecimientos recurrentes; veía en ellas unos procesos cíclicos que se propagaban como olas a lo largo del eje temporal en el continuum espacio-tiempo. Los ciclos pueden originarse en factores causales, por ejemplo movimientos planetarios, o ser modelados por la serialidad, tal como las rachas afortunadas de los jugadores. Al final de su libro Kammerer expresa su creencia de que la serialidad es

ubicua y continua en la vida, la naturaleza y el cosmos. Es el cordón umbilical que conecta pensamiento, sentimiento, ciencia y arte, con el útero del universo que les dio a luz ${ }^{26}$.

Otro gran físico cuyos pensamientos marchaban en la misma dirección fue Wolfgang Pauli, que había previsto la existencia del neutrino. Pauli compartía la creencia de Kammerer y Jung sobre los factores no-causales y no-físicos que operan en la naturaleza. Jung desarrolla su colaboración con Pauli y escriben un tratado La Sincronicidad: un principio de conexión acausal, publicado en un mismo volumen con el ensayo de Pauli sobre Kepler. Evidentemente esto tenía un significado simbólico: uno de los más grandes físicos del siglo unía fuerzas con uno de los mayores psicólogos. El resultado fue un estimulante ejercicio de especulación heterodoxa.

El tratado de Jung parte de su concepto de sincronicidad. Lo define como ocurrencia simultánea de dos acontecimientos significativos, pero no conectados causalmente. Repetición casi literal de la definición de Kammerer de serialidad como una recurrencia de cosas o acontecimientos iguales o similares en el tiempo o en el espacio.

26. Kammerer, P., Das Gesetz der Serie, Deutsche Verlags-Anstalt, Stuttgart-Berlín 1919, p. 456. 
Kammerer se constriñó a las analogías e ingenuos términos físicos, dejando de lado a la ESP (percepción extrasensorial) y las explicaciones mentalísticas, mientras que Jung se inclinó por el extremo opuesto, intentando explicar todos los fenómenos que no respondían a los términos de la causalidad física como manifestación de la mente inconsciente:

La sincronía es un fenómeno que parece conectarse esencialmente con las condiciones psiquicas; es decir, con unos procesos propios del inconsciente ${ }^{27}$.

Kammerer y Jung intentando deshacerse de las cadenas causales de la ciencia materialista, se enredan con su propia terminología, postulando un principio a-causal y luego intentando explicarlo en términos pseudocausales. Pauli reaccionó destronando la materia, la energía y la causalidad.

La revolucionaria propuesta de Pauli radicaba en extender el principio de los acontecimientos no-causales de la microfísica a la macrofísica intentando, al unir sus fuerzas a las de Jung, elaborar alguna teoría macrofísica capaz de explicar los fenómenos paranormales. El intento se frustró por culpa de tradiciones profundamente grabadas en el pensamiento occidental, que se remonta a la época de los griegos.

Como Kammerer, Jung cayó una y otra vez en espúreas explicaciones causales destinadas a poner en funcionamiento su principio a-causal. La gramática del lenguaje griego sobre el cosmos, según Sidney Hook, desbarató los afanes de Kammerer y Jung, junto a los de otros que se habían embarcado en empresas similares. La literatura de la parapsicología está llena de esperanzadas teorías que, de hecho, nacieron destinadas al fracaso.

Entre los psicólogos, Freud - una vez que se convenció de la realidad del contacto telepático entre paciente y analista - teorizó que la ESP era un método arcaico de comunicación entre los individuos, suplantado posteriormente por el medio más eficaz de la comunicación sensorial.

Entre los biólogos, sir Alister Hardy propuso una notable teoría: las actividades altamente evolucionadas y coordinadas de algunos animales inferiores, como los foraminíferos, sólo podrían ser explicadas por una suerte de mente grupal en la cual cada individuo compartiría una impronta psíquica.

Entre los filósofos, los profesores Broad y Price han presentado estimulantes hipótesis mentalísticas.

Últimamente, entre los matemáticos, G. Spencer Brown propuso una intrigante teoría que pretendía explicar los nada fortuitos resultados en los ex-

27. Jung, C.G., The Structure and Dynamics of the Psyche, Collected Works, vol. VIII, tr. Hull, R.F.C., London 1960, p. 511. 
perimentos adivinatorios de naipes, cuestionando la validez del propio concepto de casualidad.

Spencer Brown declaró que, reuniendo pares de números dígitos al azar, en los cuales el primero simbolizaba a la adivinanza y el segundo al naipeobjeto, había obtenido un número de aciertos significativamente más alto que la expectativa normal. Sin embargo no dio a publicidad sus tabulaciones, y en ningún momento declaró que sus resultados pudieran compararse, en magnitud a los promedios astronómicamente superiores a las probabilidades obtenidas por los experimentadores en ESP.

Spencer Brown admitió que los experimentos en ESP habían sido bien concebidos y rigurosamente controlados y aceptó los resultados como válidos, pero señaló que indicaban cierta anomalía en el propio concepto del azar. Aunque no elaboró su tesis sobre la naturaleza de esta presunta anomalía, que explicaría el número desproporcionadamente alto de aciertos en los experimentos ESP, sus ideas registran una aguda semejanza con el concepto de serialidad presentado por Kammerer. La ley de las series es, en realidad, recíproca al concepto de azar ${ }^{28}$.

Tanto Kammerer como Jung postulan un principio a-causal que consideran de importancia igual al de causalidad en el destino del hombre y el mundo en general.

Podemos preguntarnos para qué sirve esa agencia a-causal. La «utilidad» de la causalidad es algo que conocemos bastante bien: poner orden y estabilidad en un universo que, de otro modo, resultaría caótico e imprevisible. Causalidad significa ley y orden. Pero ¿qué significa el escarabajo de oro (Cetonia aurata) que Jung vio en su ventana precisamente cuando un paciente le relataba un sueño en el cual le entregaban un escarabajo de oro?

Desde la antigüedad hasta el siglo XVIII, los hombres tenían una respuesta pronta a esta pregunta en términos de influencias, simpatías y correspondencias.

Las constelaciones planetarias gobernaban el carácter y el destino del hombre; el macrocosmos se reflejaba en el microcosmos; todo estaba ligado, no por causas mecánicas, sino por afinidades ocultas. La doctrina de la simpatía de todas las cosas se remonta a Hipócrates quien dijo: Existe un humor común, un aliento común; todas las cosas están en simpatía ${ }^{29}$.

Este concepto se presenta como una suerte de Leit-motiv a través de las enseñanzas de los pitagóricos, los neoplatónicos y los filósofos del Renacimiento. El dualismo de la causalidad y la simpatía a-causal fue limpiamente sintetizado por el platónico Pico della Mirándola ${ }^{30}$.

28. Ver a Koestler, A., Las raíces del azar, op. cit., p. 141.

29. Cita de Koestler, A., Las raíces del azar, op. cit. p. 148.

30. Mirándola, Pico della, Opera omnia, Basle 1557, p. $40 \mathrm{f}$ 
El concepto pitagórico de armonía de las esferas, revivido por los isabelinos y la filosofía subyacente en los afanes astrólogos y alquimistas, pueden considerarse como variaciones sobre un mismo tema: las coincidencias significativas son manifestaciones de un orden universal omnicomprehensivo.

En los escritos de Kepler esta concepción se refleja en su forma más pura. La capacidad del alma humana para actuar como resonante cósmico tenía para Kepler, así como para Pico, un aspecto místico y otro causál: afirmaba la conexión del alma con el anima mindi, pero al mismo tiempo estaba sujeta a estrictas leyes geométricas.

Un siglo después Leibniz desarrolló su inmensamente influyente sistema filosófico, centrado en el concepto de la mónada. A su juicio las mónadas eran los átomos mismos de la naturaleza; pero contrariamente a los átomos materiales de Demócrito, suponían entidades espirituales, cada una de ellas un microcosmos o espejo del universo en miniatura. Las mónadas no actúan directamente entre sí, carecen de ventanas por las cuales podría entrar o salir alguna cosa, pero cada una está de "acuerdo» o "correspondencia» con todas las otras en virtud de una armonía preestablecida ${ }^{31}$.

Sólo en el siglo XVIII, alboreando la revolución newtoniana, fue entronizada la causalidad como regente absoluta de mente y materia; en las primera décadas del siglo XX la destronarían, como consecuencia de la revolución en la física.

Aún en el siglo XVIII Arthur Schopenhauer, quien ejerció gran influencia sobre Freud y Jung, proclamó que la causalidad física era uno de los rectores del mundo; el otro era una entidad metafísica, una suerte de conciencia universal, comparada con la cual la conciencia individual suponía una especie de sueño, contrapuesto al estado de vigilia.

Las teorías clásicas sobre la ESP propuestas por Carington, Tyrel, Hardy y otros eran variaciones sobre un mismo tema: el éter psíquico, o mente grupal, o inconsciente colectivo, operando como fondo subterráneo del que se alimentaban las mentes individuales, y a través del cual se comunicaban. El concepto dominante es la Unidad en la Diversidad: todo es Uno, y el Uno lo es todo.

Este aliento penetra los escritos de los místicos cristianos y constituye la piedra basal del budismo y el taoísmo. Establece los paralelos de latitud de que se hablaba en el globo de Schopenhauer, y conecta la coincidencia con un esquema universal de las cosas. Según Jung, todas las prácticas adivinatorias, desde la observación de las hojas de té hasta los complejos métodos oraculares

31. Leibniz, G., Monadologie n. 14 (tomo VI), Berlín 1885, p. 607, y Loc. cit. n. 7,10,11,15, pp. 607-609.

Véase también Monadología (traducción de Manuel Fuentes Benot), B.I.F., Aguilar, Buenos Aires 1964 (3. ${ }^{a}$ ed.) pp. 27 (n. 7), 29 (n. 11), 44-45 (n. 51) en especial. 
del I Ching, se basan en la idea de que los acontecimientos casuales son misterios menores que pueden utilizarse como indicadores con respecto al gran misterio central.

O sea, la Sincronía y la Serialidad son derivados modernos de la creencia arquetípica en la unidad fundamental de todas las cosas, superior a la causalidad mecánica.

Y efectivamente esto es así, pues podríamos comparar la evolución científica de los últimos ciento cincuenta años con un vasto sistema fluvial, donde un afluente tributario tras otro resulta devorado por la corriente principal, unificándose todos ellos en un único y anchuroso delta.

Los constituyentes últimos del universo, energía y masa, partícula y vibración, se presentaron entonces como aspectos diferentes de un mismo proceso básico, la llamada unicidad fundamental. Whitehead sintetizó la situación en forma llamativa ${ }^{32}$.

El progreso de la ciencia hacia una unidad fundamental, visible en amplia perspectiva, brinda también una analogía positiva entre el concepto de unicidad de los pitagóricos y sus descendientes, incluyendo a Kammerer y Jung, e incluso nos permite adquirir cierta tolerancia en cuanto a la obsesión de Kammerer con la idea de una atracción cuasi-gravitacional entre las entidades afines, la simbiosis, la periodicidad, la afinidad y demás.

Si Kammerer rechazó la telepatía fue sólo porque creía que aquélla establecía un misterio menor, innecesario, en relación con fenómenos que formaban parte del misterio mayor de la serialidad: la correlación universal de las cosas, su integración en una matriz universal.

Debemos hallar una unidad final compartida por el físico, el místico y el parapsicólogo; una unidad que sólo se alcanzaría a través de la diversidad, en un giro superior del espiral.

También entre los biólogos ha revivido el interés por las potencialidades de la materia viva, en cuanto a edificar formas de complejidad superior que presentan la unidad-en-la diversidad sobre un nivel más alto: en otras palabras, el interés por los poderes integradores de la vida, concepto que contrasta con la concepción de la evolución a base de mutaciones casuales.

En último análisis el azar ¿no sería sencillamente lo que no comprendo? La ventaja que podemos ver en la denominación de azar es que uno no se limita a lo antropomórfico y, en definitiva, confesamos mejor lo que no comprendemos.

El mundo vivo, tal y como lo contemplamos, incluida la raza humana, es

32. Whitenead, A.N., Nature and Life, Cambridge 1934, p. 181. 
el producto de azares filtrados por una selección. Pero quien dice selección dice prohibición. La vida tal y como se manifiesta y organiza prohíbe en cierto modo a otra cosa reproducirse... ¡Curiosa combinación de un azar que termina en algo que prohíbe el azar!... La vida y su desarrollo ¿estarían acaso dotados en cierto modo de intencionalidad? Efectivamente tenemos que evocar una idea de intencionalidad: proyecto, deseo...

El desarrollo de las ciencias modernas nos da una visión particularmente penetrante e interrogativa sobre el mundo, el mundo viviente y el hombre, y en el plano estrictamente científico la significación de este prodigioso conjunto se nos escapa totalmente. Declarar que este sentido, por escapársenos, no existe, es lo propio incluso de una gestión intelectual indebida llamada simplemente extrapolación.

Tras la experiencia del límite de las ciencias es posible otra cosa. No es por no comprender yo por lo que la cuestión desaparece, sino que es entonces cuando queda abierto a un posible inexplorable no puede ser, de orden del «saber». ¿No sería acaso en él donde aparece entonces, entre la bruma luminosa de la incertidumbre fundamental, el orden de la fe y del amor?

Antonio Masdias Quintela 